\title{
WHAT IS THE EFFECT OF SMALL SIZE URETERAL ACCESS SHEAT ON URETERAL STRICTURE DURING RETROGRADE INTRARENAL SURGERY?: A ONE-YEAR FOLLOW-UP.
}

özer güzel ${ }^{1}$, Melih Balci ${ }^{1}$, Altug Tuncel ${ }^{1}$, Ahmet Asfuroglu ${ }^{1}$, Can Aykanat ${ }^{1}$, and Yilmaz Aslan $^{1}$

${ }^{1}$ Ankara Numune Training and Research Hospital

March 5, 2021

\begin{abstract}
Aim: To analyze the ureteral injury and incidence of ureteral stricture in a series of patients who underwent retrograde intrarenal surgery with using smallest ureteral access sheath. Materials and Methods: Between September 2016 and March 2019 , 154 consecutive retrograde intrarenal surgery procedures with adjunctive use of an ureteral access sheath for kidney stone were prospectively included the study. A 9.5/11.5-F ureteral access sheath was used during procedures. The patients were evaluated in terms of intraoperative postoperative and late complications. Ureteral injuries after retrograde intrarenal surgery were assessed visually with flexible and semirigid ureterorenoscope. All patients were evaluated by computed tomographic urography in the first year after treatment for detection of ureteral stricture. Results: The mean age of the patients was $47 \pm 15$ (12-81) years. Of the patients, 86 were male and 68 were female. Mean stone size was $17.1 \pm 8$ (7-40) mm and mean operative time was $56 \pm 23$ (30-120) minutes. Overall $79.9 \%$ of patients had evidence of injury to the ureter wall. Non-significant lesion (grade 0) was seen in $39.0 \%$ of patients. Grade 1 lesions were assigned in $40.9 \%$ of patients. There were no grade 2 and higher lesions detected. A total of 5 patients $(3.2 \%)$ had minor complications. Urinary sepsis developed as a major complication in 3 patients $(1.9 \%)$. No ureteral stricture was detected in the patients at first year control. Conclusions: The results of our series indicate that the 9.5/11.5-F ureteral access sheath is safe for routine use to facilitate flexible ureteroscopy and there was no long-term adverse effect.
\end{abstract}

WHAT IS THE EFFECT OF SMALL SIZE URETERAL ACCESS SHEAT ON URETERAL STRICTURE DURING RETROGRADE INTRARENAL SURGERY?: A ONE-YEAR FOLLOW-UP.

Ozer Guzel

Melih Balci

Altug Tuncel

Ahmet Asfuroglu

İbrahim Can Aykanat

Yilmaz Aslan

University of Health Sciences, Ankara Numune Research and Training Hospital, Department of Urology, Ankara, Turkey 
Running head: Ureteral access sheath during retrograde intrarenal surgery

Correspondence to: Ozer GUZEL, M.D. Assoc. Prof.

University of Health Sciences, Ankara Numune Research and Training Hospital Department of Urology,

Talatpasa blv, No: 44, 06230, Sihhiye-Ankara/Turkey

Telephone: +905324301496

Fax: +90312312 6876

email:drozerguzel@gmail.com

\section{ABSTRACT}

Aim: To analyze the ureteral injury and incidence of ureteral stricture in a series of patients who underwent retrograde intrarenal surgery with using smallest ureteral access sheath.

Materials and Methods: Between September 2016 and March 2019, 154 consecutive retrograde intrarenal surgery procedures with adjunctive use of an ureteral access sheath for kidney stone were prospectively included the study. A 9.5/11.5-F ureteral access sheath was used during procedures. The patients were evaluated in terms of intraoperative postoperative and late complications. Ureteral injuries after retrograde intrarenal surgery were assessed visually with flexible and semirigid ureterorenoscope. All patients were evaluated by computed tomographic urography in the first year after treatment for detection of ureteral stricture.

Results: The mean age of the patients was $47 \pm 15$ (12-81) years. Of the patients, 86 were male and 68 were female. Mean stone size was $17.1 \pm 8$ (7-40) mm and mean operative time was $56 \pm 23$ (30-120) minutes. Overall $79.9 \%$ of patients had evidence of injury to the ureter wall. Non-significant lesion (grade 0) was seen in $39.0 \%$ of patients. Grade 1 lesions were assigned in $40.9 \%$ of patients. There were no grade 2 and higher lesions detected. A total of 5 patients $(3.2 \%)$ had minor complications. Urinary sepsis developed as a major complication in 3 patients (1.9\%). No ureteral stricture was detected in the patients at first year control.

Conclusions: The results of our series indicate that the 9.5/11.5-F ureteral access sheath is safe for routine use to facilitate flexible ureteroscopy and there was no long-term adverse effect.

Keywords: Ureteral access sheath, ureteral injury, complication

\section{WHAT'S KNOW?}

Parallel to the improvements in technology during past decades retrograde intrarenal surgery (RIRS) has become an efficient and safe option in the management of urinary system stone disease with a gradually increasing popularity. Ureteral access sheath (UAS) decreases intrapelvic pressure, helps with access for multiple instruments, and facilitates drainage and removal of the fragmented stones. Therefore UAS placement is a crucial step of RIRS. Although it has several advantages, UAS also remains a controversial tool in endourology due to the increased risk of ureteral injury.

\section{WHAT'S NEW?}

To our knowledge; there is no prospective study in the international literature has evaluated the effect of UAS related ureteral injury and possible ischemia on the development of ureteral stricture. In the current study, we aim to analyze intraoperative, postoperative complications and the incidence of ureteral injury with using smallest diameter UAS on the market and the effect of injuries and possible ischemia/inflammatory changes on the risk of ureteral stricture development in the one year follow-up.

\section{INTRODUCTION}

During the past decades, the surgical treatment of kidney stones has undergone many technological advances and retrograde intrarenal surgery (RIRS) became one of the standard treatments for patients with renal 
stones. The ureteral access sheath (UAS) was developed in 1974 by Hisao Takayasu\&Yoshio Aso [1] and it is often used during RIRS to facilitate entry of flexible ureteroscopes into the renal collecting system. Additionally it facilitates easy re-entry into the collecting system, consequently shortens the operating time, improve vision, decrease intrapelvic pressure and increases the flexible ureteroscope's life span [2-5]. Currently, UASs are produced with various characteristics, including various lengths, diameters, materials, dilator tip designs, radiopaque markers and stiffness [6].Selection of the UAS among the choices of manufacturers and models typically depends on physician familiarity, cost, and size of ureteroscope.

Despite their advantages, there are some important misgivings concerning to UAS use. UAS usage entails a risk of ureteral damage including involving the smooth muscle layer after insertion [7]. Additionally to the over distention created by a UAS may decrease ureteral blood flow and theoretically induce long-term ureteral stricture formation [8]. In addition to that, a previous study reported that a large sized UAS had a more effect on ureteral blood flow [9]. Therefore, a UAS with a small outer diameter and large inner diameter is considered to be ideal in clinical practice.

To our knowledge; there is no prospective study in the international literature has evaluated the effect of UAS related ureteral injury and possible ischemia on the development of ureteral stricture. In the current study, we aim to analyze intraoperative, postoperative complications and the incidence of ureteral injury with using smallest diameter UAS on the market and the effect of injuries and possible ischemia/inflammatory changes on the risk of ureteral stricture development in the long term.

\section{MATERIALS AND METHODS}

This study was approved by the local institutional ethical board (ANEAH-E-1762). All patients provided informed consent. Between September 2016 and March 2019, 154 consecutive RIRS procedures with adjunctive use of an UAS for kidney stone were prospectively included the study. All patients evaluated by anamnesis, medical history, physical examination, microscopic urinalysis, urine culture, complete blood cell count, serum biochemistry analysis, computerized tomography with/without contrast material. Patients with positive urine cultures were treated preoperatively with the appropriate antibiotics. Patients with previous stone history and urinary surgery, urinary system abnormalities (horseshoe, ectopic and malrotated kidneys, duplicated collecting systems and calyceal diverticulum stones), any degree of ureteral dilatation and the initial sheath was not placed successfully were excluded from the study. All the patients were not pre-stented.

A 9.5/11.5-F UAS (Cook, Cook Medical, Dublin, Ireland) $(35 \mathrm{~cm}$ or $45 \mathrm{~cm}$ length for females or males, respectively) was used during procedures. All procedures were performed under general anesthesia. Diagnostic ureterorenoscopy (URS) was performed by using 9.5-F semi-rigid ureteroscope (Karl Storz, Karl Storz, Tuttlingen, Germany) routinely before RIRS to detect ureteral stone or stricture, to place a hydrophilic guide-wire and for the optical dilatation. A 9.5/11.5-F ureteral access sheath was placed over the guide-wire and a 7.5-F flexible ureteroscope (Karl Storz Flex-X2, Karl Storz, Tuttlingen, Germany) was passed through the sheath. Kidney stones were fragmented with a 30W Holmium:YAG laser generator (SphinxX, Lisa, Katlenburg-Lindau, Germany). Extraction of residual fragments was not performed as a routine procedure. At the end of the operation, we entered to the ureter under guidance of guide wire with 9.5-F semi-rigid ureteroscope and whole ureter was inspected. JJ stent was inserted based on surgeon decision and removed approximately $14-28$ days postoperatively.

Intra-operative ureteral lesion grade classified using the classification methodology previously described by Traxer and Thomas[7] with categorization of five grades, ranging from 0-4, defined by the characteristics in Table 1. Modified Clavien-Dindo Classification [10] was used to report postoperative complications.

At postoperatively first month, [?] $3 \mathrm{~mm}$ residual stones were accepted as stone free. All patients were evaluated by computed tomographic urography in the first year after treatment. A stricture is defined as a fixed narrowing ureteral wall with proximal dilatation. [11]

\section{RESULTS}


The mean age of the patients was $47+-15$ (12-81) years. Of the patients, 86 were male and 68 were female. Mean stone size was $17.1+-8$ (7-40) $\mathrm{mm}$ and mean operative time was 56+-23 (30-120) minutes.

For analysis of ureteral injury, overall $79.9 \%$ of patients had evidence of injury to the ureter wall. Nonsignificant lesion (grade 0) was seen in $39 \%$ of patients. Grade 1 lesions were assigned in $40.9 \%$ of patients. No grade 2 and higher lesions were detected. In postoperative period a total of 5 patients $(3.2 \%)$ had Clavien I and II complications, including hematuria and fever. Grade IV complication developed in 3 patients $(1.9 \%)$ (Sepsis requiring intermediate care/intensive care unit management).

After follow-up period, no ureteral stricture was detected in the patients at first year control.

Immediate stone-free rate was achieved in 118 (76.6\%) patients in the first session. After first session, 30 patients underwent second RIRS session. Overall stone free rates was 90.3\% ( $\mathrm{n}=139)$. (Table 2)

\section{DISCUSSION}

The use of a UAS during RIRS, despite having several advantages over RIRS without a UAS, also which remains a controversial tool in endourology owing to an increased risk of ureteral injury. Normal ureteral lumen is narrower than any UAS on the market [12]. Insertion of a UAS dilates the ureteral wall, and thus has the risk of producing ureteral injury (variable degree mucosal erosions, mucosal and submucosal edema and hematoma) additionally placement of reinforced UAS may produce partial or even complete transection [13]. Another concern about UAS is effect on ureteral blood flow. Lallas et al. [9] investigated the possible acute ischemic effects of varying diameters UAS using a swine model. Blood flow to that segment of ureter was measured via laser doppler flowmetry and UAS remained in the ureter for 70 minutes. Authors demonstrated minimal decreases in ureteral blood flow with use of a 10/12-F (avarage; $12 \%$ ), compared with an up to $64.5 \%$ decrease with use of larger UAS. Reached the nadir blood flow averaging 20.0 to 30.0 minutes. They concluded that despite its apparent safety with regard to acute ischemic changes, one should continue to proceed with caution when selecting the appropriate-size sheath, as chronic effects remain in question. Although the reperfusion that occurs after UAS removal might expose the ureteral wall to free radicals and subsequent tissue damage [7].Another study examined the expression of the acute inflammation cytokines cyclooxygenase-2 and tumour necrosis factor alpha in ureteral tissue and demonstrated a significant 6.5fold and 8-fold upregulation of cyclooxygenase-2 and tumour necrosis factor alpha after 2 minutes of UAS deployment, respectively [14].

At first, Traxer and Thomas [7] prospectively evaluated the incidence and severity of UAS related ureteral wall injury and they generated classification system in 2013. Their study included 359 consecutive patients who underwent RIRS for kidney stone disease and 12/14-F diameter UAS was used to allow passage of the digital ureterorenoscop. The study population was divided into 2 groups, including low grade injuries (grade 0 or 1) and high grade injuries (grades 2 to 4 ), which involved the ureteral smooth muscle layers. Low grade injuries were found in $86.6 \%$ patients, grade 2 injuries observed in $10.1 \%$ patients and grade 3 injuries in $3.3 \%$. The authors did not report grade 4 injury. The incidence of postoperative complications was $7 \%$. In a recent study conducted by Loftus et al. [13]95 patients were randomized to two same size (12/14-F) different brand of UAS and they were analyzed incidence of UAS related ureteral injury. The authors used same classification system as we used [7] and they aimed to validate this 5-point classification system. End of study they found grade 1, 2, 3 and 4 injuries in $47.8 \%, 13.4 \%, 10.4 \%$ and $0.0 \%$ of patients, respectively. The authors concluded that ureteral trauma can be easily assessed using a standardized 5-point scale with good inter-rater reliability and high grade injury may be prevented by avoiding pushing against resistance when placing a sheath and using surgeon digression to switch to a smaller diameter sheath when sheath placement time is prolonged. In our series, grade 1 injury rate of $40.9 \%$ is also similar to results with two studies as mentioned above. Unlike the other two studies, we did not observe grade 2 and higher injuries. This is presumably related to our use of a smaller size of UAS. Because pushing against large size UAS into the narrow ureter during placement, possibly cause high grade ureteral trauma. Another reason may be mismatch in UAS and ureteral tone. Using the larger UAS may require more force during insertion and a higher force would mean a higher risk of ureteral injury. Koo et al. [15] found that high grade ureteral 
injury did not occur in cases in which the UAS force of insertion was $<600 \mathrm{G}(600 \mathrm{G}=5.88 \mathrm{~N})$.

We know that ureteric strictures can develop even after seemingly uncomplicated or complicated endoscopic treatment of urolithiasis. [16,17] Also decreased blood flow related to usage of UAS theoretically increase the risk of ureteral stricture. The literature also supports limiting the time of ureteroscopic procedures to avoid complications, as complications and stricture risk increase when operative time is prolonged. Loftus et al. [13]claimed that knowing the rates of ureteral stricture correlated with ureteral injury grade would help determine to what degree these injuries have clinical significance. Delvecchio et al.[8] retrospectively evaluated 62 patients undergoing 71 ureteroscopic procedures with the aid of the UAS and with complete follow-up longer than 3 months. Authors found only one stricture (1.4\%) in left ureteropelvic junction . But this patient had undergone multiple endoscopic surgeries because of recurrent struvite calculi.

In a recent study, Huang JS et al. [18] shared their data on ureteral stenosis, in which they evaluated the results of RIRS performed for kidney stones of $2 \mathrm{~cm}$ and larger. The authors reported that Over a period of 6-month follow-up, no ureteric stricture was identified. Unlike our study, Huang et al. used $13 / 15 \mathrm{~F}$ ureteral access sheaths during the operation and the follow-up times were shorter than our study. In another recent study, Sarı S et al. [19] evaluated the efficacy and safety of RIRS in 1489 patients with using UAS. The authors reported that they detected ureteral stenosis in 3 patients. In this study, 2 different size UAS $(9.5 / 11.5 \mathrm{~F}$ or $11 / 13 \mathrm{~F})$ were used and the patients were evaluated retrospectively. The major difference of our study is that it has a prospective design and we used UAS with the smallest diameter in all patients.

In the present study, we prospectively evaluated 154 consecutive RIRS procedures with adjunctive use of an UAS after 1-year follow-up and we did not detect any ureteral stricture. In spite of the fact that grade 2 or higher ureteral lesions may cause ureteral stricture because we do not detect high grade injury in our cohort. We speculate that, using larger diameter UAS may lead to more frequent ureteral injury, more ureteral ischemia, and hence more ureteral stricture. Using the smallest diameter UAS has high protection in terms of all these parameters.

There are a few limitation of our study. First of all, as we are aware that the number of patients in our study is low. We think that our study can shed light on higher volume studies. Latter; the study is of cohort design. Comparative studies can provide further contribution and information. In addition, there may be a relationship between stone types and ureteral stricture. Studies to be conducted on this subject may contribute more to the development of ureteral stenosis. Another limitation of our study is the lack of stone analysis data.

In conclusion, the results of our series indicate that the 9.5/11.5-F UAS is safe for routine use to facilitate flexible ureteroscopy and no cause complication on long term period. However, awareness of the potential ureteral wall injury and ischemic effects with the use of unnecessarily large UAS for long periods in patients at risk of injury should be considered.

\section{Acknowledgement: None}

Funding Sources: None

Statement of Ethics: This study was approved by the local institutional ethical board (ANEAH-E-1762)

Disclosure Statement: The authors have no direct or indirect commercial financial support associated with the publishing of this article.

Research involving Human Participants and/or Animals: The study was performed in accordance with the most recent version of the Declaration of Helsinki.

Informed consent: The approval of the local ethics committee was obtained and written informed consent was obtained from all the patients.

Conflicts of Interest : The authors declare that there is no conflict of interest.

\section{Author Contributions:}


Ozer Guzel: Protocol development, Manuscript writing

M Balci: Project development, Manuscript writing

A Tuncel: Manuscript editing

A Asfuroglu: Data collection

C Aykanat: Data collection

Y Aslan: Manuscript editing, Data analysis

\section{REFERENCES}

Takayasu H, Aso Y. Recent development for pyeloureteroscopy: guide tube method for its introduction into the ureter. J Urol 1974; 112:176-178.

Kourambas J, Byrne RR, Preminger GM. Does a ureteral access sheath facilitate ureteroscopy? J Urol 2001; 165:789-793.

Auge BK, Pietrow PK, Lallas CD, Raj GV, Santa-Cruz RW, Preminger GM. Ureteral access sheath provides protection against elevated renal pressures during routine flexible ureteroscopic stone manipulation. J Endourol 2004; 18:33-36.

Vanlangendonck R, Landman J. Ureteral access strategies: pro-access sheath. Urol Clin North Am 2004; 31:71-81.

Rehman J, Monga M, Landman J et al. Characterization of intrapelvic pressure during ureteropyeloscopy with ureteral access sheaths. Urology 2003; 61:713-718.

De Coninck V, Keller EX, Rodríguez-Monsalve M, Audouin M, Doizi S, Traxer O. Systematic review of ureteral access sheaths: facts and myths. BJU Int 2018; 122:959-969.

Traxer O, Thomas A. Prospective Evaluation and Classification of Ureteral Wall Injuries Resulting from Insertion of a Ureteral Access Sheath During Retrograde Intrarenal Surgery. J Urol 2013; 189:580-584.

Delvecchio FC, Auge BK, Brizuela RM, Weizer AZ, Silverstein AD, Lallas CD, et al. Assessment of stricture formation with the ureteral access sheath. Urology 2003; 61:518-522.

Lallas CD, Auge BK, Raj GV, Madden JF, Preminger GM. Laser Doppler flowmetric determination of ureteral blood flow after ureteral access sheath placement. J Endourol 2002; 16:583-590.

Dindo D, Demartines N, Clavien PA. Classification of surgical complications: a new proposal with evaluation in a cohort of 6336 patients and results of a survey. Ann Surg 2004; 240: 205-13.

Shokeir AA, El-Diasty T, Eassa W, Mosbah A, El-Ghar MA, Mansour O et al. Diagnosis of ureteral obstruction in patients with compromised renal function: the role of noninvasive imaging modalities. J Urol 2004; 171:2303-6.

Zelenko N, Coll D, Rosenfeld AT, Smith RC. Normal ureter size on unenhanced helical CT. AJR Am J Roentgenol 2004; 182:1039-1041.

Loftus CJ, Ganesan V, Traxer O, Schold JD, Noble M, Sivalingam S, et al. Ureteral Wall Injury with Ureteral Access Sheaths: A Randomized Prospective Trial. J Endourol 2020; 34:932-936.

Lildal SK, Norregaard R, Andreassen KH, Christiansen FE, Jung H, Pedersen MR, et al. Ureteral Access Sheath Influence on the Ureteral Wall Evaluated by Cyclooxygenase-2 and Tumor Necrosis Factor-alpha in a Porcine Model. J Endourol 2017; 31:307-313.

Koo KC, Yoon JH, Park NC, Lee HS, Ahn HK, Lee KS, et al. The Impact of Preoperative $\alpha$-Adrenergic Antagonists on Ureteral Access Sheath Insertion Force and the Upper Limit of Force Required to Avoid Ureteral Mucosal Injury: A Randomized Controlled Study. J Urol 2018; 199:1622-1630. 
Engel O, Rink M, Fisch M. Management of iatrogenic ureteral injury and techniques for ureteral reconstruction. Curr Opin Urol 2015; 25:331-5.

May PC, Hsi RS, Tran H, Stoller ML, Chew BH, Chi T, et al. The Morbidity of Ureteral Strictures in Patients with Prior Ureteroscopic Stone Surgery: Multi-Institutional Outcomes. J Endourol 2018; 32:309-314.

Huang JS, Xie J, Huang XJ, Yuan Q, Jiang HT, Xiao KF. Flexible ureteroscopy and laser lithotripsy for renal stones $2 \mathrm{~cm}$ or greater: A single institutional experience. Medicine (Baltimore) 2020; 23;99:e22704.

Sari S, Cakici MC, Aykac A, Baran O, Selmi V, Karakoyunlu AN. Outcomes with ureteral access sheath in retrograde intrarenal surgery: a retrospective comparative analysis. Ann Saudi Med 2020; 40:382-388.

\section{Hosted file}

Table 1.pdf available at https://authorea.com/users/399717/articles/512174-what-is-theeffect-of-small-size-ureteral-access-sheat-on-ureteral-stricture-during-retrogradeintrarenal-surgery-a-one-year-follow-up

\section{Hosted file}

Table 2.pdf available at https://authorea.com/users/399717/articles/512174-what-is-theeffect-of-small-size-ureteral-access-sheat-on-ureteral-stricture-during-retrogradeintrarenal-surgery-a-one-year-follow-up 